\title{
Multiple-line Chemotherapy for a Patient with Unresectable Mucinous Cystic Neoplasm of the Pancreas
}

\author{
Haruo Miwa ${ }^{1}$, Kazuya Sugimori ${ }^{1}$, Tomohiro Ishii ${ }^{1}$, Akihiro Funaoka ${ }^{1}$, Hiromi Tsuchiya ${ }^{1}$, \\ Yoshimasa Suzuki ${ }^{1}$, Makoto Sugimori ${ }^{1}$, Masaki Nishimura ${ }^{1}$, Yuichiro Tozuka ${ }^{1}$, \\ Satoshi Komiyama ${ }^{1}$, Takeshi Sato ${ }^{1}$, Takashi Kaneko ${ }^{1}$, Kazushi Numata ${ }^{1}$ and Shin Maeda ${ }^{2}$
}

\begin{abstract}
:
A 74-year-old woman with a cyst in her pancreatic tail was referred to our hospital. Computed tomography confirmed a large cystic lesion with irregular wall thickening, abdominal lymph node swelling, and ascites. We diagnosed her with an unresectable mucinous cystic neoplasm, since ascites cytology revealed adenocarcinoma. The patient received chemotherapy up to the fifth line for 55.2 months. Gemcitabine plus nabpaclitaxel and modified FOLFIRINOX achieved a partial response with a progression-free survival time of 12.1 and 20.4 months, respectively. The overall survival time from the beginning of first-line chemotherapy was 69.4 months.
\end{abstract}

Key words: antineoplastic combined chemotherapy protocols, palliative therapy, pancreatic cancer, pancreatic cyst

(Intern Med 60: 2607-2612, 2021)

(DOI: 10.2169/internalmedicine.6755-20)

\section{Introduction}

Mucinous cystic neoplasms (MCNs) are rare disease, representing about $20 \%$ of all resected pancreatic cysts, and are known to occur in the pancreatic body and tail in women $(1,2)$. Although MCNs are considered to have malignant potential, unresectable cases due to metastases or local invasion have rarely been reported. Palliative chemotherapy has been recommended for unresectable $\mathrm{MCN}$ in some reports $(3,4)$; however, no clinical evidence has been established about the efficacy of such treatment.

In recent years, the efficacy of palliative chemotherapy for metastatic pancreatic ductal adenocarcinoma (PDAC) has evolved. Combination regimens, such as FOLFIRINOX or gemcitabine plus nab-paclitaxel $(\mathrm{GnP})$, have been reported to achieve a high response rates and an improved overall survival $(5,6)$. However, there are few reports on the efficacy of these combined chemotherapy regimens in unresectable MCN (7).
We herein report a patient with unresectable MCN who achieved a long-term survival under multi-line chemotherapy.

\section{Case Report}

A 74-year-old woman with a cystic lesion in her abdominal cavity was referred to the Yokohama City University Medical Center. During the physical examination, a soft, fist-sized mass was palpable in her left upper abdomen. Her serum tumor marker levels were normal (carcinoembryonic antigen: $3.6 \mathrm{ng} / \mathrm{mL}$ and carbohydrate antigen 19-9: $29 \mathrm{U} /$ $\mathrm{mL}$ ). Contrast-enhanced computed tomography (CECT) showed a cystic lesion of $9 \mathrm{~cm}$ in diameter with enhanced irregular wall thickening in the pancreatic tail (Fig. 1). There were enlarged lymph nodes along the hepatic artery (No. 12a) and on the anterior surface of the pancreas (No. 17). Ascites and tiny nodules around the mesentery were also observed. Contrast-enhanced transabdominal ultrasonography showed an enhanced septum inside the cyst

${ }^{1}$ Gastroenterological Center, Yokohama City University Medical Center, Japan and ${ }^{2}$ Division of Gastroenterology, Yokohama City University Graduate School of Medicine, Japan

Received: November 19, 2020; Accepted: January 14, 2021; Advance Publication by J-STAGE: March 1, 2021

Correspondence to Dr. Haruo Miwa, miwa@yokohama-cu.ac.jp 

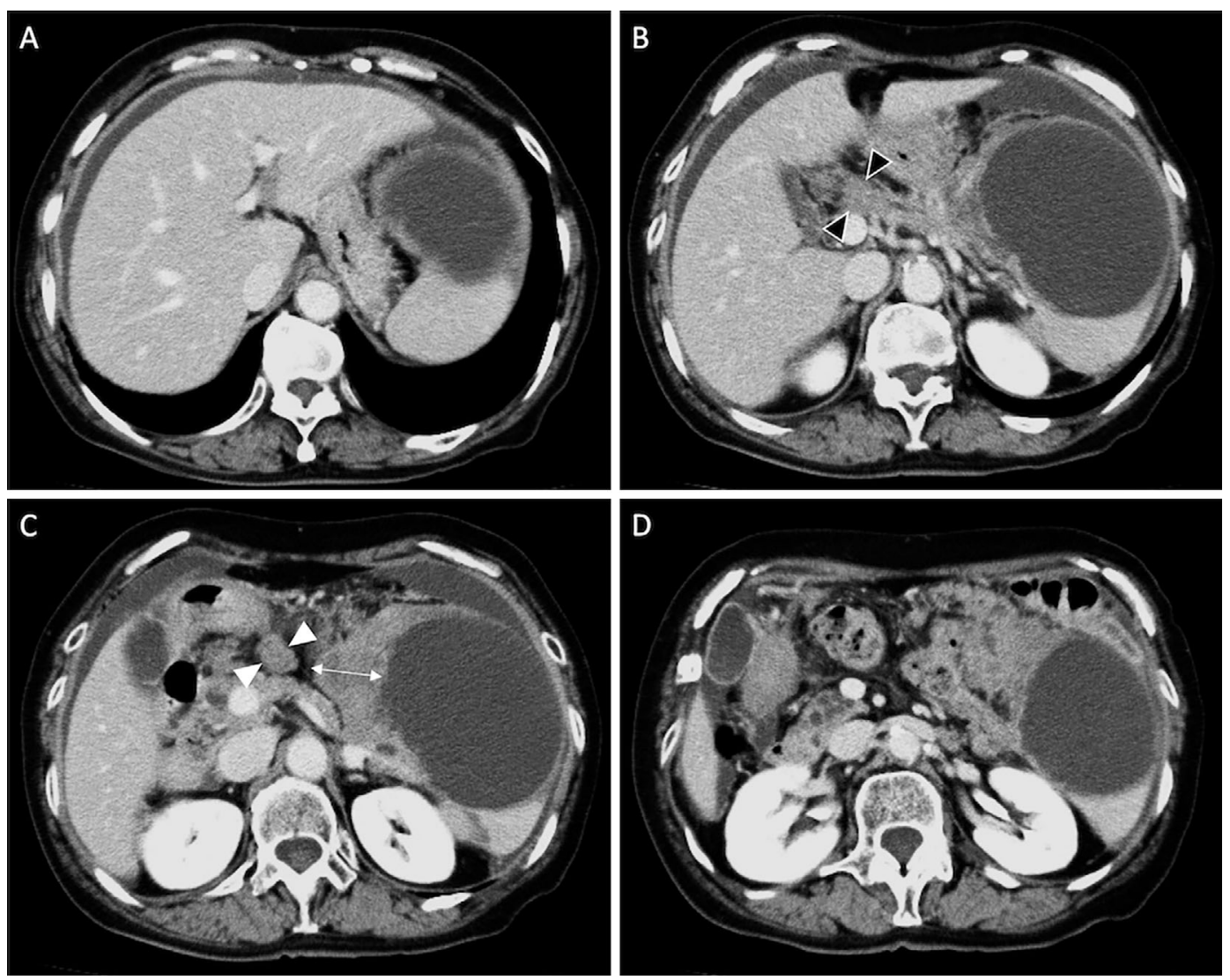

Figure 1. A 74-year-old woman with a mucinous cystic neoplasm of the pancreas. Contrast-enhanced computed tomography shows a 9-cm cystic lesion in the pancreas tail. Wall thickening is visible at the right side of the cyst (arrow). Swollen lymph nodes can be observed along the hepatic artery (black arrowhead) and in front of the pancreatic body (white arrowhead). Ascites is present around the liver and spleen.
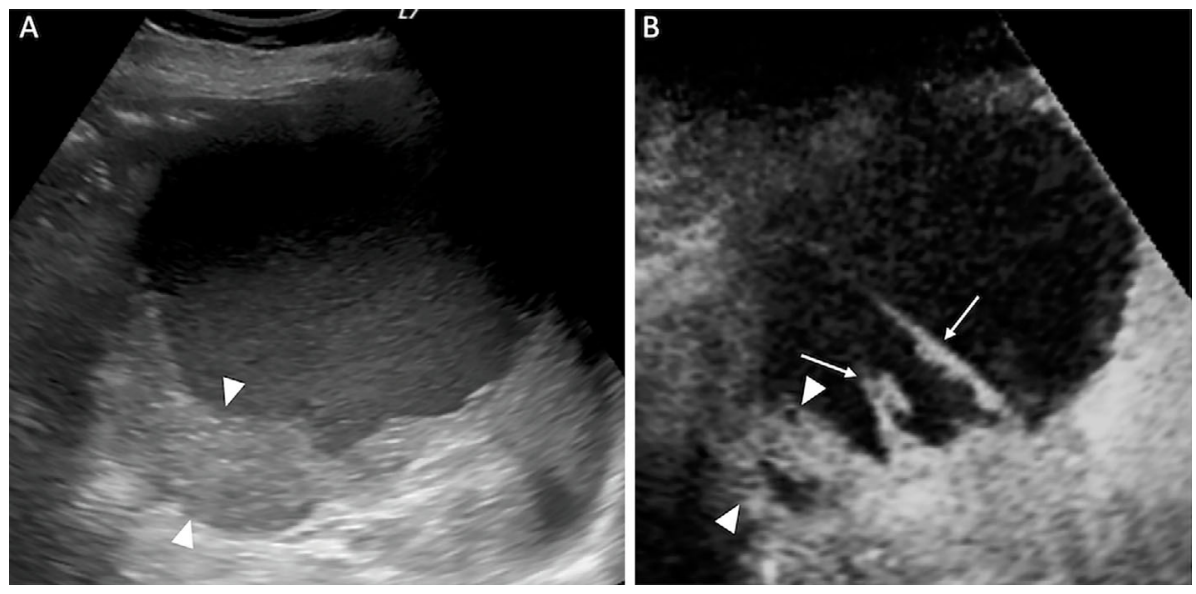

Figure 2. A) Conventional transabdominal ultrasound shows a cystic lesion with wall thickening and hyperechoic content. B) Contrast-enhanced transabdominal ultrasonography reveals an enhanced septum inside the cyst (arrows). The thickened wall shows blood flow (arrowheads).

(Fig. 2). Endoscopic ultrasonography did not reveal a connection between the cyst and the main pancreatic duct. Ascites cytology revealed an adenocarcinoma (Fig. 3); therefore, we made a diagnosis of MCN with an associated invasive carcinoma with peritoneal dissemination and lymph node metastases. We considered palliative chemotherapy to be the best option for the patient.

First-line chemotherapy consisting of $1,000 \mathrm{mg} / \mathrm{m}^{2} \mathrm{gem}-$ citabine (GEM) on day 1, 8, and 15 every 4 weeks was started. At the beginning of treatment, the patient's performance status (PS) according to the Eastern Cooperative Oncology Group (ECOG) was Grade 0. After 3.9 months, 


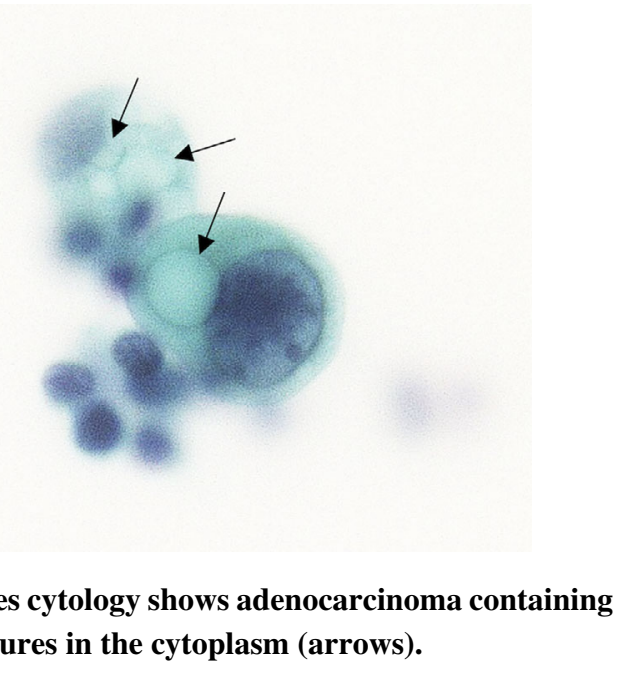

mucus-like structures in the cytoplasm (arrows).

CECT showed enlarged lymph nodes, indicating progressive disease (PD) based on the Response Evaluation Criteria in Solid Tumors (RECIST). The regimen was changed to 100 $\mathrm{mg} /$ day of $\mathrm{S}-1$, an oral fluoropyrimidine, for 2 weeks, followed by a 1 -week washout period. All adverse events were graded according to the Common Terminology Criteria for Adverse Events, Version 4.0. The patient suffered from nausea and fatigue (Grade 2). After 6.6 months, the lymph nodes were again enlarged on CECT. Regarding GEM and S-1 monotherapy, the best effect of chemotherapy achieved was stable disease (SD), and the progression-free survival was 3.9 and 6.6 months, while the average relative dose intensity (ARDI) was $100 \%$ and $88 \%$, respectively.

Subsequently, third-line chemotherapy was administered with $125 \mathrm{mg} / \mathrm{m}^{2}$ of nab-paclitaxel plus $1,000 \mathrm{mg} / \mathrm{m}^{2}$ of GEM on day 1,8 , and 15 repeated every 4 weeks. Because of neutropenia (Grade 3), the dose of both nab-paclitaxel and GEM was reduced to $60 \%$ of the initial dose, and the administration schedule was extended to biweekly. After 7.6 months, CECT showed significant improvement of the wall thickening of the cyst, shrinkage of the lymph nodes, and disappearance of ascites, which were considered to reflect a partial response (PR). GnP was continued for 20 cycles until PD was diagnosed based on exacerbation of the wall thickening of the cyst. The progression free survival (PFS) was 12.1 months, and the ARDI was $40 \%$.

Since the patient's general condition was good at an ECOG-PS of 0, we selected modified FOLFIRINOX as the regimen for fourth-line chemotherapy, consisting of $65 \mathrm{mg}$ / $\mathrm{m}^{2}$ of oxaliplatin, $120 \mathrm{mg} / \mathrm{m}^{2}$ of irinotecan, and $300 \mathrm{mg} / \mathrm{m}^{2}$ of leucovorin, followed by a continuous infusion of 2,400 $\mathrm{mg} / \mathrm{m}^{2}$ of 5 -FU over a 46 -hour period, every 2 weeks. The dosage of oxaliplatin and irinotecan was reduced to $80 \%$ of the initial dose because of the elderly age of the patient. She experienced fatigue and peripheral sensory neuropathy, assessed as Grade 2, until cycle 2. Both oxaliplatin and irinotecan were reduced to $60 \%$ of the initial dose by the ninth cycle. After 1.9 months (3 cycles), a PR was achieved with remarkable shrinkage of the lymph nodes and improvement of wall thickening of the cyst. Modified FOLFIRINOX was continued with treatment interruption for three months and one month because of heart failure (Grade 3) and herpes zoster (Grade 2), respectively. After 20 cycles, CECT showed progression of the wall thickening of the cyst and enlargement of the lymph nodes, which were considered to reflect PD. The PFS under modified FOLFIRINOX was the longest among all regimens at 20.1 months. The ARDI of the 3 drugs - oxaliplatin, irinotecan, and 5-FU - was 35\% (Fig. 4).

We suggested the patient finish palliative chemotherapy; however, she strongly preferred to continue anti-cancer therapy. Therefore, fifth-line chemotherapy with $1,000 \mathrm{mg} / \mathrm{m}^{2}$ of GEM on day 1 and $80 \mathrm{mg} /$ day of S-1 from day 1 to 7 was administered every 3 weeks, and the PFS was 5.1 months. After 9.4 months, best supportive care was provided, but the ascites increased, and her general condition deteriorated to ECOG PS 2.

Another seven months later, the patient was hospitalized for one week due to an infection inside the cyst, but conservative therapy with antibiotics was effective in resolving this. Eventually, she died as a consequence of the primary tumor after 14.1 months of best supportive care. The overall survival from the beginning of first-line chemotherapy was 69.4 months, including 55.2 months receiving palliative chemotherapy (Table) (Fig. 5).

\section{Discussion}

MCN of the pancreas is a rare tumor that occurs predominantly in middle-aged women (8). When diagnosing MCN, it is important to differentiate it from branched-type intraductal papillary mucinous neoplasm (IPMN) (9). MCN is histologically diagnosed based on the existence of an ovarian-type stroma $(10,11)$. If a surgical specimen is not available, the clinical diagnosis is made based on the characteristics of a unilocular or septated macrocystic lesion without connection to the pancreatic duct, enhancing mural nodules on CECT, and wall thickening $(12,13)$. A cytological analysis after endoscopic ultrasound-guided fine-needle aspiration can detect malignant cystic lesions. However, it is difficult to differentiate between IPMN and MCN with a small specimen (14). We did not aspirate any contents of the cyst; however, the cytology of the patient's ascites revealed an adenocarcinoma. In the present case, the diagnosis of MCN was confirmed by the characteristics of a cyst with irregular wall thickening and no connection to the main pancreatic duct.

Although MCN is considered to have malignant potential, there are only a few reports describing the rate of malignancy. Postlewait et al. (2), reported that $14.9 \%$ of 349 patients with resected pancreatic MCN had adenocarcinoma or high-grade dysplasia. Among them, lymph node metastasis was observed in about one-third. Due to this low incidence of metastasis, the prognosis of malignant MCN was better than that of PDAC, with a 3-year survival rate of $59 \%$. 


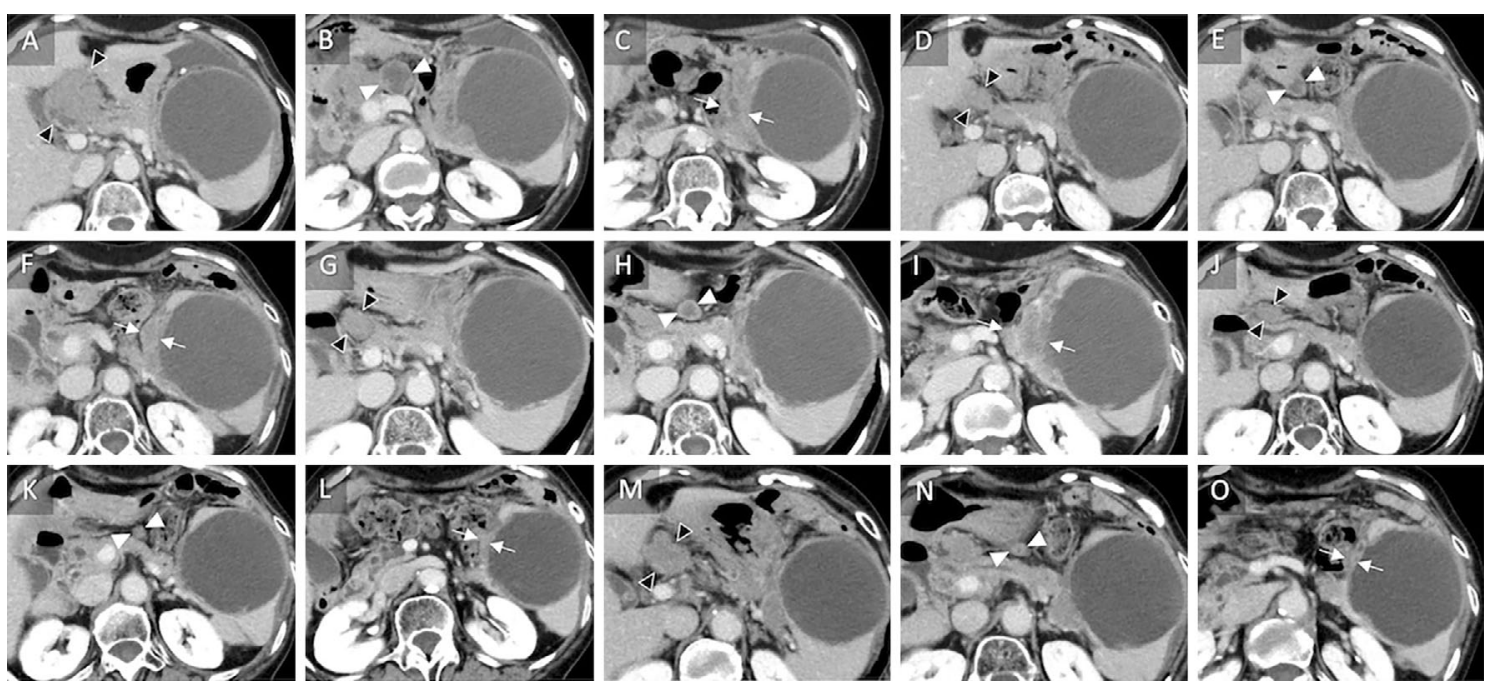

Figure 4. Sequential computed tomography images obtained over 2.5 years during combined chemotherapy of a mucinous cystic neoplasm of the pancreas. A, B, C) At the start of nab-paclitaxel plus gemcitabine as third-line chemotherapy, the lymph nodes along the hilar artery (black arrowhead) and anterior portion of the pancreas (white arrowhead) and wall thickening of the cyst (arrows) are shown. D, E, F) A partial response is achieved after six cycles (seven months) of nab-paclitaxel plus gemcitabine with the findings of lymph nodes shrinkage. G, H, I) After 20 cycles (13 months), enlargement of the lymph nodes along the hilar artery (black arrowheads) and wall thickening (arrows) confirm progressive disease. $\mathrm{J}, \mathrm{K}, \mathrm{L}$ ) A partial response is visible after three cycles (two months) of modified FOLFIRINOX. M, N, O) After 20 cycles (20.4 month), the enlargement of the lymph nodes along the hilar artery (black arrowheads) demonstrates progressive disease.

Table. Results of Systematic Chemotherapy.

\begin{tabular}{lcccc}
\hline & Regimen & Best Response & PFS & ARDI \\
\hline 1st line & Gemcitabine & SD & 3.9 months & $100 \%$ \\
2nd line & S-1 & SD & 6.6 months & $88 \%$ \\
3rd line & Nab-paclitaxel plus Gemcitabine & PR & 12.1 months & $40 \%$ \\
4th line & Modified-FOLFIRINOX & PR & 20.4 months & $35 \%$ \\
5th line & Gemcitabine plus S-1 & SD & 5.1 months & $44 \%$ \\
\hline
\end{tabular}

Effect of chemotherapy was evaluated by RECIST guideline. FOLFIRINOX is consist of oxaliplatin, irinotecan, 5-FU, and leucovorin. RECIST: response evaluation criteria in solid tumors, PFS: progression free survival, ARDI: average relative dose intensity, PR: partial response, SD: stable disease

However, regarding unresectable $\mathrm{MCN}$, the prognosis is significantly worse than that of surgically treated cases $(8,15,16)$.

Although palliative chemotherapy is recommended for unresectable or recurrent cystic lesions of the pancreas (14), there is currently no evidence guiding the best choice of regimen for unresectable MCN. Werner et al. (4). reported 5 patients who received palliative chemotherapy for unresectable MCN and observed a median survival time of 11 months (range: 4-37 months). In that report, GEM-based treatment had been selected in accordance with the recommended palliative chemotherapy of PDAC. However, the outcomes of chemotherapy for PDAC have radically improved with the advent of two regimens: FOLFIRINOX and $\mathrm{GnP}(5,6)$. These combined chemotherapies have resulted in remarkable increases in the survival compared with GEM alone in patients with metastatic PDAC. However, these regimens also increase the frequency of severe adverse events; therefore, they are not recommended for use in patients with a poor general condition or an old age. At the time of initiating treatment for the present patient, $\mathrm{GnP}$ was not yet covered by national health insurance in Japan, and we did not have adequate experience regarding the clinical use of FOLFIRINOX. In addition, there were no reports concerning the use of $\mathrm{GnP}$ or FOLFIRINOX for MCN at the time. Therefore, we selected a less toxic monotherapy of GEM or S-1 as the first and second regimens. However, neither of these monotherapies achieved tumor shrinkage, so we switched to more toxic regimens.

Although FOLFIRINOX and GnP have been reported to be highly toxic, we were able to administer both of them to our patient for long periods of time at reduced dosages. For 


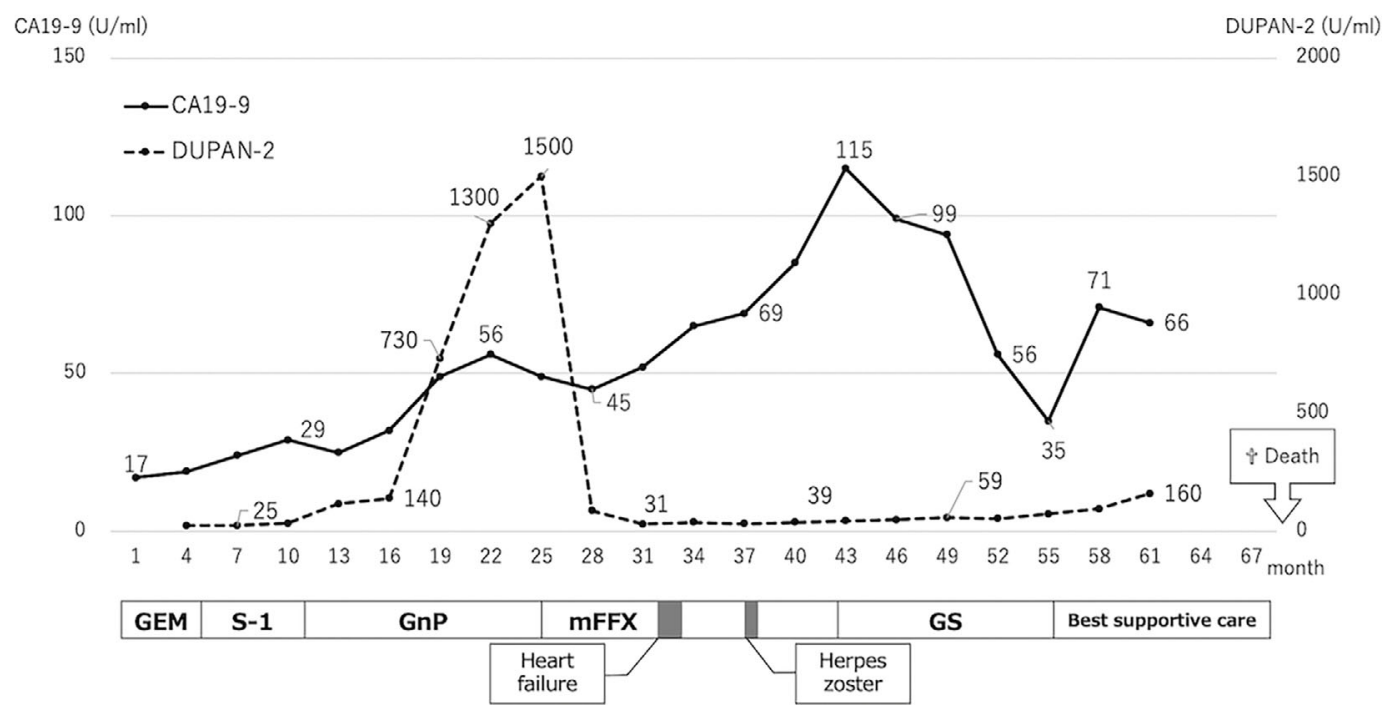

Figure 5. Clinical course with changes in tumor markers and transition for each regimen. GEM: gemcitabine, GnP: nab-paclitaxel plus gemcitabine, mFFX: Modified-FOLFIRINOX, GS: gemcitabine plus $S-1$

$\mathrm{GnP}$, we reduced the dosage and frequency because of neutropenia (Grade 3) in the fourth cycle. However, after the fifth cycle, no severe adverse events were observed. The ARDI of GnP was lower than the values noted in previous reports $(6,17)$; however, our patient was able to continue treatment for up to 12.1 months while maintaining a good PS. Brunetti et al. (7) reported the overall survival time of patients with unresectable MCN treated with GnP and FOLFIRINOX as 13 months and 9-14 months, respectively. Among these patients, 2 received both regimens, and their overall survival times were 9 and 14 months, respectively. Compared with these cases, the present case achieved a long survival.

Despite the fact that our patient has already received GEM monotherapy, GnP achieved PR, with the antitumor effect lasting for one year. This result may be due to the fact that nab-paclitaxel increases intratumoral GEM levels. Frese et al. (18) reported that paclitaxel reduced the levels of cytidine deaminase protein, which is associated with the metabolization of GEM, in mice. This synergistic effect may be the reason for the tumor reduction under GnP, although the ARDI was as low as $40 \%$.

It may be considered controversial to administer modified FOLFIRINOX as the fourth-line chemotherapy because the patient was 76 years old at the time. Furthermore, there were no clinical reports that modified FOLFIRINOX was effective in patients with unresectable MCN. However, the patient's good general condition (ECOG-PS 0) and her strong preference supported the decision to choose modified FOLFIRINOX. After three cycles, the thickness of the cyst wall had remarkably regressed on CECT, and the regimen was continued with intermissions for 20 cycles. Modified FOLFIRINOX had to be stopped because of heart failure (Grade 3 ) for 3 months after 8 cycles and because of herpes zoster (Grade 2) for 1 month after 10 cycles. However, we were able to restart the same regimen with dose reductions.

Several studies investigated the efficacy of modified FOLFIRINOX in patients with GEM-refractory PDAC (19-21). Sawada et al. reported the efficacy and good tolerability of modified FOLFIRINOX as second-line therapy after GnP (22). Although FOLFIRINOX poses a risk of serious adverse events, these studies have shown that it can be safely administered when doses are reduced. In this case, both oxaliplatin and irinotecan were first reduced to $80 \%$ of the initial dose and ultimately to $60 \%$. Considering the interruptions in the administration, the ARDI reached 35\%. Nevertheless, modified FOLFIRINOX was continued without tumor progression for 20.4 months. Peripheral neuropathy is the most important adverse event associated with the crossover therapy of GnP and modified FOLFIRINOX. Our patient suffered Grade 2 peripheral neuropathy in the course of modified FOLFIRINOX that did not require treatment interruption.

In conclusion, unresectable $\mathrm{MCN}$ is rare, and there is little clinical evidence supporting its treatment with combined chemotherapy. In our patient, only $\mathrm{GnP}$ and modified FOLFIRINOX achieved PR among the five regimens used. Although further large-scale prospective studies are required to confirm the efficacy and feasibility in patients with unresectable MCN, we recommend GnP and modified FOLFIRINOX to prolong the survival under palliative chemotherapy.

The authors state that they have no Conflict of Interest (COI).

\section{References}

1. Nilsson LN, Keane MG, Shamali A, et al. Nature and management of pancreatic mucinous cystic neoplasm (MCN): a systematic review of the literature. Pancreatology 16: 1028-1036, 2016.

2. Postlewait LM, Ethun CG, McInnis MR, et al. Association of pre- 
operative risk factors with malignancy in pancreatic mucinous cystic neoplasms: a multicenter study. JAMA Surg 152: 19-25, 2017.

3. Shimada K, Iwase K, Aono T, et al. A case of advanced mucinous cystadenocarcinoma of the pancreas with peritoneal dissemination responding to gemcitabine. Gan To Kagaku Ryoho 36: 995-998, 2009.

4. Björk Werner J, Sturesson C, Dawiskiba S, Andersson R. Mucinous cystadenocarcinoma of the pancreas - outcome following different modes of treatment. Ann Gastroenterol 24: 213-217, 2011.

5. Conroy T, Desseigne F, Ychou M, et al. FOLFIRINOX versus gemcitabine for metastatic pancreatic cancer. N Engl J Med 364: 1817-1825, 2011.

6. Von Hoff DD, Ervin T, Arena FP, et al. Increased survival in pancreatic cancer with nab-paclitaxel plus gemcitabine. N Engl J Med 369: 1691-1703, 2013.

7. Brunetti O, Aprile G, Marchetti P, et al. Systemic chemotherapy for advanced rare pancreatic histotype tumors: a retrospective multicenter analysis. Pancreas 47: 759-771, 2018.

8. Le Borgne J, de Calan L, Partensky C. Cystadenomas and cystadenocarcinomas of the pancreas: a multiinstitutional retrospective study of 398 cases. French Surgical Association. Ann Surg 230 152-161, 1999.

9. Crippa S, Fernandez-Del Castillo C, Salvia R, et al. Mucinproducing neoplasms of the pancreas: an analysis of distinguishing clinical and epidemiologic characteristics. Clin Gastroenterol Hepatol 8: 213-219, 2010.

10. Tanaka M, Fernandez-del Castillo $C$, Adsay V, et al. International consensus guidelines 2012 for the management of IPMN and MCN of the pancreas. Pancreatology 12: 183-197, 2012.

11. Goh BK, Tan YM, Chung YF, et al. A review of mucinous cystic neoplasms of the pancreas defined by ovarian-type stroma: clinicopathological features of 344 patients. World J Surg 30: 2236-2245, 2006.

12. Scholten L, van Huijgevoort NCM, van Hooft JE, Besselink MG, Del Chiaro M. Pancreatic cystic neoplasms: different types, different management, new guidelines. Visc Med 34: 173-177, 2018.

13. Garces-Descovich A, Beker K, Castillo-Angeles M, et al. Mucinous cystic neoplasms of the pancreas: high-resolution crosssectional imaging features with clinico-pathologic correlation. $\mathrm{Ab}-$ dom Radiol (NY) 43: 1413-1422, 2018.

14. European Study Group on Cystic Tumours of the P. European evidence-based guidelines on pancreatic cystic neoplasms. Gut 67: 789-804, 2018.

15. Suzuki Y, Atomi Y, Sugiyama M, et al. Cystic neoplasm of the pancreas: a Japanese multiinstitutional study of intraductal papillary mucinous tumor and mucinous cystic tumor. Pancreas 28: 241-246, 2004.

16. Doulamis IP, Mylonas KS, Kalfountzos CE, Mou D, Haj-Ibrahim H, Nasioudis D. Pancreatic mucinous cystadenocarcinoma: epidemiology and outcomes. Int J Surg 35: 76-82, 2016.

17. Ueno $H$, Ikeda $M$, Ueno $M$, et al. Phase I/II study of nabpaclitaxel plus gemcitabine for chemotherapy-naive Japanese patients with metastatic pancreatic cancer. Cancer Chemother Pharmacol 77: 595-603, 2016.

18. Frese KK, Neesse A, Cook N, et al. Nab-paclitaxel potentiates gemcitabine activity by reducing cytidine deaminase levels in a mouse model of pancreatic cancer. Cancer Discov 2: 260-269, 2012.

19. Kobayashi N, Shimamura T, Tokuhisa M, Goto A, Endo I, Ichikawa Y. Effect of FOLFIRINOX as second-line chemotherapy for metastatic pancreatic cancer after gemcitabine-based chemotherapy failure. Medicine (Baltimore) 96: e6769, 2017.

20. Kim JH, Lee SC, Oh SY, et al. Attenuated FOLFIRINOX in the salvage treatment of gemcitabine-refractory advanced pancreatic cancer: a phase II study. Cancer Commun (London) 38: 32, 2018.

21. Chung MJ, Kang H, Kim HG, et al. Multicenter phase II trial of modified FOLFIRINOX in gemcitabine-refractory pancreatic cancer. World J Gastrointest Oncol 10: 505-515, 2018.

22. Sawada M, Kasuga A, Mie T, et al. Modified FOLFIRINOX as a second-line therapy following gemcitabine plus nab-paclitaxel therapy in metastatic pancreatic cancer. BMC Cancer 20: 449, 2020.

The Internal Medicine is an Open Access journal distributed under the Creative Commons Attribution-NonCommercial-NoDerivatives 4.0 International License. To view the details of this license, please visit (https://creativecommons.org/licenses/ by-nc-nd/4.0/).

(C) 2021 The Japanese Society of Internal Medicine Intern Med 60: 2607-2612, 2021 